\title{
The gut microbiota in the common kestrel (Falco tinnunculus): a report from the Beijing Raptor Rescue Center
}

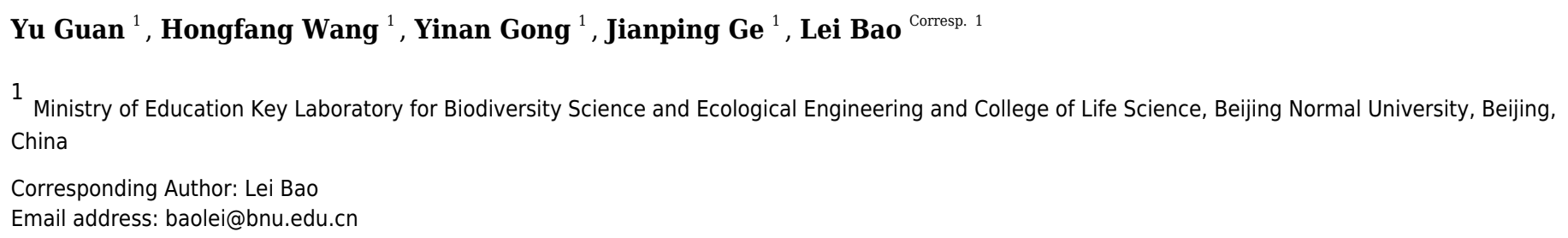

As a complex microecological system, the gut microbiota plays crucial roles in many aspects, including immunology, physiology and development. The specific function and mechanism of the gut microbiota in birds are distinct due to their body structure, physiological attributes and life history. Data on the gut microbiota of the common kestrel, a second-class protected animal species in China, are currently scarce. With highthroughput sequencing technology, we characterized the bacterial community of the gut from 9 fecal samples from a wounded common kestrel by sequencing the V3-V4 region of the $16 \mathrm{~S}$ ribosomal RNA gene. Our results showed that Proteobacteria (41.078\%), Firmicutes (40.923\%) and Actinobacteria (11.191\%) were the most predominant phyla. Lactobacillus (20.563\%) was the most dominant genus, followed by Escherichia-Shigella (17.588\%) and Acinetobacter (5.956\%). Our results would offer fundamental data and direction for the wildlife rescue. 
1 The Gut Microbiota in the Common Kestrel (Falco tinnunculus): a

2 Report from the Beijing Raptor Rescue Center

3

4 Yu Guan, Hongfang Wang, Yinan Gong, Jianping Ge, Lei Bao*

5

6 Ministry of Education Key Laboratory for Biodiversity Science and Ecological Engineering and

7 College of Life Science, Beijing Normal University, Beijing 100875, China

9 E-mail addresses:

10 Yu Guan: guanyu1990@hotmail.com

11 Hongfang Wang: wanghf@,bnu.edu.cn

12 Yinan Gong: gongyinan07@163.com

13 Jianping Ge: gejp@,bnu.edu.cn

14 Lei Bao*: baolei@,bnu.edu.cn

15 * Corresponding author at: No. 19, Xinjiekouwai Street, Haidian District, Beijing 100875, P. R.

16 China. Tel.: 86(10) 58804806.

17 


\section{Abstract}

As a complex microecological system, the gut microbiota plays crucial roles in many aspects, including immunology, physiology and development. The specific function and mechanism of the gut microbiota in birds are distinct due to their body structure, physiological attributes and life history. Data on the gut microbiota of the common kestrel, a second-class protected animal species in China, are currently scarce. With high-throughput sequencing technology, we characterized the bacterial community of the gut from 9 fecal samples from a wounded common kestrel by sequencing the V3-V4 region of the 16S ribosomal RNA gene. Our results showed that Proteobacteria (41.078\%), Firmicutes (40.923\%) and Actinobacteria (11.191\%) were the most predominant phyla. Lactobacillus $(20.563 \%)$ was the most dominant genus, followed by Escherichia-Shigella (17.588\%) and Acinetobacter (5.956\%). Our results would offer fundamental data and direction for the wildlife rescue.

Keywords: Common kestrel (Falco tinnunculus), Gut microbiota, 16S rRNA gene, Highthroughput sequencing, Noninvasive

\section{Introduction}

Recent research on host-associated gut microbial communities have revealed their important roles in immunology, physiology and development (Guarner \& Malagelada 2003; Nicholson et al. 2005), as well as several basic and critical processes, such as nutrient absorption and vitamins synthesis in both human and animals (Fukuda \& Ohno 2014; Kau et al. 2011; Omahony et al. 2015). Gut microbiota analysis of wild animals is becoming a new method that may provide information for wildlife rescue and animal husbandry. Reports concerning the gut microbiota of 
53 other avian species, such as Cooper's hawk (Accipiter cooperii) (Taylor et al. 2019), bar-headed

54 geese (Anser indicus) (Wang et al. 2017), hooded crane (Grus monacha) (Zhao et al. 2017),

55 Western Gull (Larus occidentalis) (Cockerham et al. 2019), herring gull (Larus argentatus)

56 (Fuirst et al. 2018) and black-legged kittiwake (Rissa tridactyla) (van Dongen et al. 2013), have

57 increased rapidly. The specific function and mechanism of the gut microbiota in birds are distinct

58 due to their body structure, physiological attributes and life history (Kobayashi 1969; Williams

$59 \&$ Tieleman 2005; Winter et al. 2006). For example, for most birds, a stable body temperature

60 above ambient temperature ensures a high metabolic rate for the birds needed for flight (O'Mara

61 et al. 2017; Schleucher 2002; Smit et al. 2016). Streamlined bodies, efficient breathing patterns

62 and relatively short gastrointestinal tracts are also special attributes (Klasing 1999; Orosz \&

63 Lichtenberger 2011). Meanwhile, the birds' ability to fly sets them apart from other animals,

64 altering their intestinal microbiota to some extent. However, as a research focus, data on the gut

65 microbiota of the common kestrel are currently very scarce.

66 The common kestrel (Falco tinnunculus) is a small raptor that belongs to Falconidae, which is a

67 family of diurnal birds of prey, including falcons and kestrels. A total of 12 subspecies for

68 common kestrel are distributed widely from the Palearctic to Oriental regions (Cramp \& Brooks

69 1992). Although listed in the least concern (LC) class by the International Union for

70 Conservation of Nature (IUCN) (BirdLife International. 2016), the common kestrel was listed

71 as state second-class protected animals (Defined by the LAW OF THE PEOPLE'S REPUBLIC

72 OF CHINA ON THE PROTECTION OF WILDLIFE, Chapter II, Article 9) in China. The

73 common kestrel is a typical opportunistic forager that catches small and medium-sized animals,

74 including small mammals, birds, reptiles and some invertebrates (Anthony 1993; Aparicio 2000;

75 Village 2010). Insects such as grasshoppers and dragonflies were also identified in the diet of the

76 common kestrel (Geng et al. 2009). As generalist predators, common kestrels choose distinct

77 predatory strategies when non-breeding and breeding to minimize the expenditure of energy, 
such as the strategy of the low-cost low-profit technique of perch-hunting in winter, while maximized daily energy gain in summer (Costantini et al. 2005; Masman et al. 1988). Previous studies on common kestrels were comprehensive, such as those on diet and prey selection (Geng et al. 2009; Kirkwood 1980; Korpimäki 1985; Lihu et al. 2007; Souttou et al. 2007; Van Zyl 1994), behavior and diseases (Aschwanden et al. 2005; Bustamante 1994; Hille et al. 2007), and genetic variation and diversity (Nesje et al. 2000; Padilla et al. 2009; Riegert et al. 2010; Zhang et al. 2008). As common raptors around the whole world, as well as the important predators in food chains, common kestrels should be studied more deeply with the newer methods and techniques.

The aim of this study was to characterize the bacterial community of the gut by sequencing the V3-V4 region of the 16S rRNA gene of a wounded common kestrel. The data we obtained could provide basic information for further conservation and rescue of wild common kestrels.

\section{Materials \& Methods}

Fecal samples collection

This study is of a single kestrel sampled multiple times with feces at Beijing Raptor Rescue Center (BRRC). The injured common kestrel that could not fly was found in the Fengtai district by a rescuer on June 22nd, 2019 and then taken to the BRRC for professional rescue. The wounded common kestrel was carefully treated with several surgeries and drug therapies. Nine fecal samples (E1-E9) that may reflect the actual state of its health were collected from the common kestrel after relevant treatments on different days. The samples collection information and medical records of the common kestrel were shown in Table S1-S3 respectively. All samples were transported immediately into the laboratory in an ice box and ultimately stored at $-80^{\circ} \mathrm{C}$ for further bacterial studies.

DNA extraction and PCR amplification 
104 Microbial DNA was extracted from fresh fecal samples using an E.Z.N.A.® Stool DNA Kit

105 (Omega Bio-tek, Norcross, GA, U.S.) according to the manufacturer's protocols. The V3-V4

106 region of the bacterial $16 \mathrm{~S}$ ribosomal RNA gene was amplified by $\mathrm{PCR}\left(95^{\circ} \mathrm{C}\right.$ for 3 min;

107 followed by 25 cycles at $95{ }^{\circ} \mathrm{C}$ for $30 \mathrm{~s}, 55^{\circ} \mathrm{C}$ for $30 \mathrm{~s}$, and $72{ }^{\circ} \mathrm{C}$ for $30 \mathrm{~s}$; and a final extension

108 at $72{ }^{\circ} \mathrm{C}$ for $5 \mathrm{~min}$ ) using the primers 338F (5'-barcode-ACTCCTACGGGAGGCAGCAG-3')

109 and 806R (5'-GGACTACHVGGGTWTCTAAT-3'), where the barcode is an eight-base

110 sequence unique to each sample. PCRs were performed in triplicate in a $20 \mu \mathrm{L}$ mixture

111 containing $4 \mu \mathrm{L}$ of $5 \times$ FastPfu Buffer, $2 \mu \mathrm{L}$ of $2.5 \mathrm{mM}$ dNTPs, $0.8 \mu \mathrm{L}$ of each primer $(5 \mu \mathrm{M})$,

$1120.4 \mu \mathrm{L}$ of FastPfu Polymerase, and $10 \mathrm{ng}$ of template DNA.

113

114 Illumina MiSeq sequencing

115 Amplicons were extracted from 2\% agarose gels and purified using an AxyPrep DNA Gel

116 Extraction Kit (Axygen Biosciences, Union City, CA, U.S.) according to the manufacturer's

117 instructions and quantified using QuantiFluor ${ }^{\mathrm{TM}}$-ST (Promega, U.S.). Purified amplicons were

118 pooled in equimolar amounts and paired-end sequenced $(2 \times 250)$ on an Illumina MiSeq platform

119 according to standard protocols.

120

121 Processing of sequencing data

122 Raw fastq files were demultiplexed and quality-filtered using QIIME (version 1.17) (Caporaso et

123 al. 2010) with the following criteria. (i) The $300 \mathrm{bp}$ reads were truncated at any site receiving an

124 average quality score $<20$ over a $50 \mathrm{bp}$ sliding window, discarding the truncated reads that were

125 shorter than 50 bp. (ii) Exact barcode matching, 2 nucleotide mismatches in primer matching,

126 and reads containing ambiguous characters were removed. (iii) Only sequences that overlapped

127 longer than $10 \mathrm{bp}$ were assembled according to their overlap sequence. Reads that could not be

128 assembled were discarded. 
129 Operational taxonomic units (OTUs) were clustered with a 97\% similarity cutoff using UPARSE

130 (version 7.1 http://drive5.com/uparse/), and chimeric sequences were identified and removed

131 using UCHIME (Edgar et al. 2011). The taxonomy of each 16S rRNA gene sequence was

132 analyzed by RDP Classifier (http://rdp.cme.msu.edu/) against the SILVA (SSU115)16S rRNA

133 database using a confidence threshold of 70\% (Amato et al. 2013).

134

135 Data analysis

136 All the indices of alpha diversity, including Chao, ACE, Shannon, Simpson, and coverage, and

137 the analysis of beta diversity were calculated with QIIME. The rarefaction curves, rank

138 abundance curves, and stacked histogram of relative abundance were displayed with R (R Core

139 Team, 2015).

140 The hierarchical clustering trees were built using UPGMA (unweighted pair-group method with

141 arithmetic mean) based on weighted and unweighted distance matrices at different levels.

142 Principal coordinate analysis (PCoA) was calculated and displayed using QIIME and R, as well

143 as hierarchical clustering trees.

144 This study was performed in accordance with the recommendations of the Animal Ethics Review

145 Committee of Beijing Normal University (approval reference number: CLS-EAW-2019-026).

146

\section{Results}

148 Overall sequencing data

149 A total of 28 phyla, 70 classes, 183 orders, 329 families and 681 genera were detected among the

150 gastrointestinal bacterial communities. There were altogether 389,474 reads obtained and

151 classified into 1673 OTUs at the 0.97 sequence identity cut-off in 9 fecal samples from a

152 common kestrel.

153 Alpha diversity indices (including Sobs, Shannon, Simpson, ACE, Chao and coverage) of each

154 sample are shown in Table 1. The Sobs and Shannon index of all samples are shown in Fig. 1. 
155 Additionally, the rarefaction curves (A) and the rank abundance curves (B) are shown in Fig. S1,

156 which indicated that the number of OTUs for further analysis was reasonable, as well as the

157 abundance of species in common kestrel feces. The total sequences, total bases and OTU

158 distributions of all samples are shown in Table S4 and Table S5.

159

160 Bacterial composition and relative abundance

161 At the phylum level of the gut microbiota in the common kestrel, the most predominant phylum

162 was Proteobacteria (41.078\%), followed by Firmicutes (40.923\%), Actinobacteria (11.191\%)

163 and Bacteroidetes (3.821\%). In addition to Tenericutes $(0.178 \%)$ and Verrucomicrobia

164 (0.162\%), Patescibacteria (0.543\%) and Deinococcus-Thermus $(0.504 \%)$ were also ranked in the

165 top 10 species in the common kestrel fecal microbiota (Table 2).

166 The top 5 families in the gut microbiota were Lactobacillaceae (20.563\%), Enterobacteriaceae

167 (18.346\%), Moraxellaceae (6.733\%), Bifidobacteriaceae (5.624\%) and Burkholderiaceae

$168(4.752 \%)$.

169 At the genus level, Lactobacillus (20.563\%), Escherichia-Shigella (17.588\%) and Acinetobacter

$170 \quad(5.956 \%)$ were the most dominant genera. These were followed by Bifidobacterium (5.624\%)

171 and Enterococcus (4.024\%) (Table 3). These five genera in the total gut microbiota of several

172 samples accounted for a small proportion, such as for E5 (28.755\%) and E6 (10.905\%) and

173 especially for E4 (2.861\%), while the largest proportion was $98.416 \%$ in E1.

174 The stacked histogram of relative abundance for species is also demonstrated in Fig. 2 at the

175 phylum (A) and genus (B) levels, which could intuitively represent the basic bacterial

176 composition and relative abundance. The community structures of E1 and E9 were more similar

177 than those of the other feces samples at both levels.

178 The hierarchical clustering trees showed the similarity of community structure among different

179 samples, which were generated by UPGMA (unweighted pair-group method with arithmetic

180 mean) with the unweighted UniFrac (Fig. 3A) and weighted UniFrac (Fig. 3B) distance matrixes. 
181 Although the fecal samples were collected from the common kestrel in chronological order (E1-

182 E9) of therapy treatments, no distinct or obvious clustering relationships are discernable in Fig.

1833.

184

185 Discrepancy of community composition

186 To further demonstrate the differences in community composition among the nine samples,

187 principal coordinates analysis (PCoA) was applied (Fig. 4). For PCoA, we chose the same two

188 distance matrices (unweighted UniFrac in Fig. $4 A$ and weighted UniFrac in Fig. 4B) as above to

189 analyze the discrepancies. The results in Fig. 4 were similar to those in Fig. 3, in which all

190 samples scattered dispersedly, suggesting that variation in the composition of the gut microbiota

191 of the common kestrel was not obvious over time.

192

193 Discussion

194 Knowledge and comprehension concerning gut microbiota have continued to progressively

195 develop with relevant techniques over the past decade (Guarner 2014; Li et al. 2014; Qin et al.

196 2010). The application of analysis for intestinal microecology continues to be also a research

197 focus in the field of wildlife rescue.

198 The common kestrel (Falco tinnunculus) is listed as a second-class protected animal species in

199 China. Although research concerning avian species, including the common kestrel, has been

200 increasing gradually, the available data on the gut microbiota in the common kestrel were

201 currently unknown.

202 We characterized the basic composition and structure of the gut microbiota from a wounded

203 common kestrel in this study, which was rescued by the Beijing Raptor Rescue Center (BRRC).

204 In general, the overall community structure of the gut microbiota in this common kestrel was in

205 accordance with previous relevant characterizations in birds, such as Cooper's hawks (Taylor et

206 al. 2019), bar-headed geese (Wang et al. 2017), hooded cranes (Zhao et al. 2017) and swan geese 
207 (Wang et al. 2016), which included Proteobacteria, Firmicutes, Actinobacteria and

208 Bacteroidetes.

209 The most predominant phylum in the fecal gut microbiota of the common kestrel was

210 Proteobacteria (41.078\%), which ranked after Firmicutes in other birds, such as cockatiels

211 (Nymphicus hollandicus) (Alcaraz et al. 2016) and black-legged kittiwakes (van Dongen et al.

212 2013). This crucial phylum plays many valuable roles. For instance, Proteobacteria is beneficial

213 for the giant panda, which can degrade lignin in its major food resource (Fang et al. 2012).

214 Additionally, it has been reported that Proteobacteria is also the most dominant phylum in obese

215 dogs (Park et al. 2015). The specific function of this phylum could be distinct in birds due to

216 their unique physiological traits, as well as their developmental strategies (Kohl 2012). However,

217 the high relative abundance of Proteobacteria in the total bacterial community was observed

218 mainly in several samples that were collected during surgeries or drug treatments, such as E1 and

219 E4. Sample E1 was collected on 23rd June that the day after the kestrel rescued from the wild.

220 On 22nd June, the kestrel was bandaged with silver sulfadiazine cream (SSD), also

221 subcutaneously injected with $10 \mathrm{ml}$ and orally administered with $4 \mathrm{ml}$ lactated ringer's solution

222 (LRS) respectively. The increased level of Proteobacteria was associated with some

223 cardiovascular events, inflammation and inflammatory bowel disease (Amar et al. 2013;

224 Carvalho et al. 2012). Although the kestrel's weight increased 34 grams when E4 was collected,

225 it just ate a mouse's head. Combined with the status when the kestrel was rescued, we speculated

226 that the increased proportion of Proteobacteria may reflect its food consumption or

227 gastrointestinal status to some extent. Environmental influential factors, as well as dietary

228 changes, should also be considered an important index that could result in variations in the

229 relative abundance of species in the gut microbiota (De Filippo et al. 2010; Scott et al. 2013).

230 Furthermore, the dominant genera within Proteobacteria in our study were Escherichia-Shigella

231 (17.588\%), Acinetobacter (5.956\%), Paracoccus (2.904\%) and Burkholderia-Caballeronia-

232 Paraburkholderia (2.408\%). Escherichia-Shigella is a common pathogenic bacterium that can 
233 cause diarrhea in humans (Hermes et al. 2009). The main cause for the high relative abundance

234 of Escherichia-Shigella was the E1 (88.610\%) sample, which suggested indirectly that the

235 physical condition of the common kestrel was not normal when it was rescued by staff from the

236 BRRC. This result was also consistent with the actual state of this wounded common kestrel that

237 we observed (Table S3).

238 Although Firmicutes (40.923\%) ranked after Proteobacteria, its actual relative abundance was

239 only slightly lower than that in the common kestrel. As a common phylum of the gut microbiota,

240 Firmicutes exists widely in both mammals and birds, and this ancient symbiosis may be linked to

241 the common ancestor of amniotes (Costello et al. 2010; Kohl 2012). Firmicutes can provide

242 certain energy for the host through catabolizing complex carbohydrates, sugar, and even by

243 digesting fiber in some species (Costa et al. 2012; Flint et al. 2008; Guan et al. 2017).

244 The dominant genera in Firmicutes were Lactobacillus (20.563\%), Enterococcus (4.024\%) and

245 Clostridium_sensu_stricto_1 (3.586\%). The relative abundance of Enterococcus in E5

$246(15.026 \%)$ contributed to the highest ranking of this genus. Enterococcus is not regarded as a

247 pathogenic bacterium due to its harmlessness and can even be used as a normal food additive in

248 related industries (Fisher \& Phillips 2009; Moreno et al. 2006). Enterococcus species are also

249 considered common nosocomial pathogens that can cause a high death rate (Lopes et al. 2005).

250 Meanwhile, these species are also associated with certain infections, including neonatal

251 infections, intraabdominal and pelvic infections, as well as the nosocomial infections and

252 superinfections (Murray 1990). Coincidentally, prior to the collection of sample E5, the kestrel

253 was anesthetized for the treatment of the right tarsometatarsus injury. The right digit tendon of

254 the kestrel was exposed before managing the wound, without any function. Although ensuring

255 the sterile conditions, we inferred that the kestrel was infected by certain bacteria during the

256 surgery. The BRRC could be regarded as a specific hospital for raptor, which could explain the

257 high proportion of Enterococcus in the fecal samples of this common kestrel. However, this

258 genus should be given sufficient attention in subsequent studies with additional samples from 
259

260

261

262

263

264

265

266

267

268

269

270

271

272

273

274

275

276

277

278

279

280

281

282

283

284

different individuals. The abundance of Clostridium increases as more protein is digested (Lubbs et al. 2009). Clostridium difficile has been reported to be associated with certain diseases, such as diarrhea and severely life-threatening pseudomembranous colitis (Kuijper et al. 2006; Pepin et al. 2004). The high relative abundance of this genus also resulted primarily from certain samples (E8, 28.177\%), similar to the Enterococcus mentioned above. And it's remarkable that the collection of sample E8 was in the same situation as E5. On 13th July, the kestrel also underwent surgery under anesthesia. While E5 was collected, the kestrel's status was still normal according to relevant records. These results indicated that the high relative abundance of certain pathogens may not show any symptoms of illness for the kestrel. In general, the abnormal situation of E5 and E8 still need to be paid enough attention. Moreover, to minimize the influences due to the individual differences, more samples from different individuals should be collected for further study.

The third dominant phylum in the gut microbiota in our study was Actinobacteria (11.191\%), which was also detected in other species, such as turkeys (Meleagris gallopavo) (Wilkinson et al. 2017) and Leach's storm petrel (Oceanodroma leucorhoa) (Pearce et al. 2017). The relative abundance of Actinobacteria varied in different species, such as house cats (7.30\%) and dogs (1.8\%) (Handl et al. 2011), but only accounted for $0.53 \%$ in wolves (Wu et al. 2017). Within this phylum, Bifidobacterium (5.624\%) and Glutamicibacter (1.840\%) were the primary genera. The presence of Bifidobacterium is closely related to the utilization of glycans produced by the host, as well as oligosaccharides in human milk (Sela et al. 2008; Turroni et al. 2010). Noticeably, Bifidobacterium thermophilum was reported to be used through oral administration for chickens to resist E. coli infection (Kobayashi et al. 2002). The detection and application of Bifidobacterium, especially for the rescue of many rare avian species, would be worth considering for curing various diseases in the future.

Additionally, the relative abundance of Bacteroidetes was $3.821 \%$ in this study, which consisted mainly of Sphingobacterium. Bacteroidetes is another important component of the gut 
285

286

287

288

289

290

291

292

293

294

295

296

297

298

299

300

301

302

303

304

305

306

307

308

309

310

microbiota that can degrade relevant carbohydrates from secretions of the gut, as well as high molecular weight substances (Thoetkiattikul et al. 2013). The proportion of Bacteroidetes, which was stable in most samples we collected except E5 (18.166\%), would increase correspondingly with weight loss for mice or changes in fiber content in rural children's daily diet (De Filippo et al. 2010; Ley et al. 2006; Turnbaugh et al. 2008). However, the weight of the kestrel was increasing during the collection of E5 and E8. Additionally, although the kestrel underwent surgery on 4th July, the reason for the high proportion of Bacteroidetes in its fecal sample E5 were unclear. To characterize the basic composition and structure of the gut microbiota for the common kestrel more accurately, additional fresh fecal samples from healthy individuals should be collected in follow-up studies.

Furthermore, additional attention should be paid to the high ranking of Patescibacteria $(0.543 \%)$ and Deinococcus-Thermus $(0.504 \%)$ at the phylum level. Patescibacteria might be related to basic biosynthesis of amino acids, nucleotides and so on (Lemos et al. 2019). Members of Deinococcus-Thermus are known mainly for their capability to resist extreme radiation, including ultraviolet radiation, as well as oxidizing agents (Cox \& Battista 2005; Griffiths \& Gupta 2007). The specific function of certain species in these phyla for the common kestrel should be studied by controlled experiments, detailed observations or more advanced approaches, as molecular biological techniques are developed.

In addition to the quantity of samples, living environment, age, sex and individual differentiation should also be considered as influencing factors, which would cause a degree of discrepancies at all levels in the gut microbiota. In addition, A comparison of wounded and healthy samples for the bacterial composition in the intestinal microbiota is another essential research direction that may provide additional information for wild animal rescue, such as important biomarkers that indirectly indicate potential diseases.

\section{Conclusion}


311 In summary, using high-throughput sequencing technology in this study, we first characterized

312 the elementary bacterial composition and structure of the gut microbiota for a wounded common

313 kestrel in the BRRC, which could provide valuable basic data for future studies. Further research

314 on Enterococcus, Patescibacteria and Deinococcus-Thermus should be conducted in the future

315 with additional samples. The integration of other auxiliary techniques or disciplines, such as

316 metagenomics and transcriptomics, could offer a deeper understanding of the function and

317 mechanism of the gut microbiota, as well as the wildlife rescue.

318

319 Acknowledgments

320 We sincerely thank the Beijing Raptor Rescue Center (BRRC) and Professor Limin Feng for 321 sample collection of from the common kestrel.

322

323

\section{References}

324 BirdLife International. 2016. Falco tinnunculus. The IUCN Red List of Threatened Species 2016: e.T22696362A93556429. https://dx.doi.org/10.2305/IUCN.UK.20163.RLTS.T22696362A93556429.en. Downloaded on 09 August 2020.

R Core Team, 2015. R: A language and environment for statistical computing. R Foundation for Statistical Computing, Vienna, Austria. http://www.R-project.org/.

Alcaraz LD, Hernández AM, and Peimbert M. 2016. Exploring the cockatiel (Nymphicus hollandicus) fecal microbiome, bacterial inhabitants of a worldwide pet. PeerJ 4:e2837.

Amar J, Lange C, Payros G, Garret C, Chabo C, Lantieri O, Courtney M, Marre M, Charles M, Balkau B, 332 and Burcelin R. 2013. Blood Microbiota Dysbiosis Is Associated with the Onset of Cardiovascular Events in a Large General Population: The D.E.S.I.R. Study. PloS One 8(1): e54461. https://doi.org/10.1371/journal.pone.0054461. 
Amato KR, Yeoman CJ, Kent A, Righini N, Carbonero F, Estrada A, Gaskins HR, Stumpf RM, Yildirim S, and Torralba M. 2013. Habitat degradation impacts black howler monkey (Alouatta pigra) gastrointestinal microbiomes. ISME J 7:1344.

Anthony V. 1993. Foraging of the South African Rock Kestrel (Falco tinnunculus rupicolus). Biology and conservation of small falcons: Hawk and Owl Trust London, 151-162.

Aparicio JM. 2000. Differences in the diets of resident and non-resident Kestrels in Spain. Ornis Fennica 77:169-175.

Aschwanden J, Birrer S, and Jenni L. 2005. Are ecological compensation areas attractive hunting sites for common kestrels (Falco tinnunculus) and long-eared owls (Asio otus)? J Ornithol 146:279-286.

Bustamante J. 1994. Behavior of colonial common kestrels (Falco tinnunculus) during the post-fledging dependence period in southwestern Spain. J Raptor Res 28:79-83.

Caporaso JG, Kuczynski J, Stombaugh J, Bittinger K, Bushman FD, Costello EK, Fierer N, Pena AG, Goodrich JK, and Gordon JI. 2010. QIIME allows analysis of high-throughput community sequencing data. Nature Methods 7:335.

Carvalho FA, Koren O, Goodrich JK, Johansson MEV, Nalbantoglu I, Aitken JD, Su Y, Chassaing B, Walters WA, and Gonzalez A. 2012. Transient Inability to Manage Proteobacteria Promotes Chronic Gut Inflammation in TLR5-Deficient Mice. Cell Host \& Microbe 12:139-152.

Cockerham S, Lee B, Orben R, Suryan R, Torres L, Warzybok P, Bradley R, Jahncke J, Young H, Ouverney C, and Shaffer S. 2019. Microbial Ecology of the Western Gull (Larus occidentalis). Microbial Ecology 78(3):665-676.

Costa MC, Arroyo LG, Allen-Vercoe E, Stämpfli HR, Kim PT, Sturgeon A, and Weese JS. 2012. Comparison of the fecal microbiota of healthy horses and horses with colitis by high throughput sequencing of the V3-V5 region of the 16S rRNA gene. PloS One 7:e41484.

Costantini D, Casagrande S, Di Lieto G, Fanfani A, and Dell'Omo G. 2005. Consistent differences in feeding habits between neighbouring breeding kestrels. Behaviour:1403-1415. 
360

361

362

363

364

365

366

367

368

369

370

371

372

373

374

375

376

377

378

379

380

381

382

383

384

385

Costello EK, Gordon JI, Secor SM, and Knight R. 2010. Postprandial remodeling of the gut microbiota in Burmese pythons. ISME $J$ 4:1375.

Cox MM, and Battista JR. 2005. Deinococcus radiodurans - the consummate survivor. Nature Reviews: Microbiology 3:882.

Cramp S, and Brooks D. 1992. Handbook of the birds of Europe, the Middle East and North Africa. The birds of the western Palearctic, vol. VI. Warblers: Oxford university Press, Oxford.

De Filippo C, Cavalieri D, Di Paola M, Ramazzotti M, Poullet JB, Massart S, Collini S, Pieraccini G, and Lionetti P. 2010. Impact of diet in shaping gut microbiota revealed by a comparative study in children from Europe and rural Africa. P Natl Acad Sci USA 107:14691-14696.

Edgar RC, Haas BJ, Clemente JC, Quince C, and Knight R. 2011. UCHIME improves sensitivity and speed of chimera detection. Bioinformatics 27:2194-2200.

Fang W, Fang Z, Zhou P, Chang F, Hong Y, Zhang X, Peng H, and Xiao Y. 2012. Evidence for lignin oxidation by the giant panda fecal microbiome. PloS One 7:e50312.

Fisher K, and Phillips C. 2009. The ecology, epidemiology and virulence of Enterococcus. Microbiology 155:1749-1757.

Flint HJ, Bayer EA, Rincon MT, Lamed R, and White BA. 2008. Polysaccharide utilization by gut bacteria: potential for new insights from genomic analysis. Nature Reviews: Microbiology 6:121.

Fuirst M, Veit RR, Hahn M, Dheilly NM, and Thorne LH. 2018. Effects of urbanization on the foraging ecology and microbiota of the generalist seabird Larus argentatus. PloS One 13(12): e0209200. https://doi.org/10.1371/journal.pone.0209200.

Fukuda S, and Ohno H. 2014. Gut microbiome and metabolic diseases. Seminars in Immunopathology 36:103-114.

Geng R, Zhang X, Ou W, Sun H, Lei F, Gao W, and Wang H. 2009. Diet and prey consumption of breeding Common Kestrel (Falco tinnunculus) in Northeast China. Prog Nat Sci 19:1501-1507.

Griffiths E, and Gupta RS. 2007. Identification of signature proteins that are distinctive of the Deinococcus-Thermus phylum. International Microbiology 10:201. 
386

387

388

389

390

391

392

393

394

395

396

397

398

399

400

401

402

403

404

405

406

407

408

409

410

Guan Y, Yang H, Han S, Feng L, Wang T, and Ge J. 2017. Comparison of the gut microbiota composition between wild and captive sika deer (Cervus nippon hortulorum) from feces by highthroughput sequencing. AMB Express 7:212.

Guarner F. 2014. Decade in review_gut microbiota: The gut microbiota era marches on. Nature Reviews Gastroenterology \& Hepatology 11:647-649.

Guarner F, and Malagelada J-R. 2003. Gut flora in health and disease. The Lancet 361:512-519.

Handl S, Dowd SE, Garcia-Mazcorro JF, Steiner JM, and Suchodolski JS. 2011. Massive parallel 16S rRNA gene pyrosequencing reveals highly diverse fecal bacterial and fungal communities in healthy dogs and cats. FEMS Microbiology Ecology 76:301-310.

Hermes RG, Molist F, Ywazaki M, Nofrarías M, Gomez de Segura A, Gasa J, and Pérez J. 2009. Effect of dietary level of protein and fiber on the productive performance and health status of piglets. Journal of Animal Science 87:3569-3577.

Hille SM, Nash JP, and Krone O. 2007. Hematozoa in endemic subspecies of common kestrel in the Cape Verde Islands. Journal of Wildlife Diseases 43:752-757.

Kau AL, Ahern PP, Griffin NW, Goodman AL, and Gordon JI. 2011. Human nutrition, the gut microbiome and the immune system. Nature 474:327-336.

Kirkwood JK. 1980. Management of a colony of common kestrels (Falco tinnunculus) in captivity. Laboratory Animals 14:313-316.

Klasing KC. 1999. Avian gastrointestinal anatomy and physiology. Seminars in Avian and Exotic Pet Medicine 8:42-50. https://doi.org/10.1016/S1055-937X(99)80036-X

Kobayashi C, Yokoyama H, Nguyen SV, Hashi T, Kuroki M, and Kodama Y. 2002. Enhancement of chicken resistance against Escherichia coli infection by oral administration of Bifidobacterium thermophilum preparations. Avian Diseases 46:542-546.

Kobayashi S. 1969. On the fine structure of the carotid body of the bird, Uroloncha domestica. Archives of Histology and Cytology 31:9-19. 
411 Kohl KD. 2012. Diversity and function of the avian gut microbiota. Journal of Comparative Physiology B: Biochemical, Systemic, and Environmental Physiology 182:591-602.

413 Korpimäki E. 1985. Diet of the Kestrel Falco tinnunculus in the breeding season. Ornis Fennica 62:130137.

Kuijper EJ, Coignard B, and Tull P. 2006. Emergence of Clostridium difficile-associated disease in North America and Europe. Clinical Microbiology and Infection 12:2-18.

Lemos LN, Medeiros JD, Dini-Andreote F, Fernandes GR, Varani AM, Oliveira G, and Pylro VS. 2019. Genomic signatures and co-occurrence patterns of the ultra-small Saccharimonadia (phylum CPR/Patescibacteria) suggest a symbiotic lifestyle. Molecular Ecology 28:4259-4271.

Ley RE, Turnbaugh PJ, Klein S, and Gordon JI. 2006. Microbial ecology: human gut microbes associated with obesity. Nature 444:1022.

Li J, Jia H, Cai X, Zhong H, Feng Q, Sunagawa S, Arumugam M, Kultima JR, Prifti E, and Nielsen T. 2014. An integrated catalog of reference genes in the human gut microbiome. Nature Biotechnology 32:834-841.

Lihu X, Jianjian L, Chunfu T, and Wenshan H. 2007. Foraging area and hunting technique selection of 426 Common Kestrel (Falco tinnunculus) in winter: the role of perch sites. Acta Ecologica Sinica $27: 2160-2166$.

Lopes MdFS, Ribeiro T, Abrantes M, Marques JJF, Tenreiro R, and Crespo MTB. 2005. Antimicrobial resistance profiles of dairy and clinical isolates and type strains of enterococci. International Journal of Food Microbiology 103:191-198.

Lubbs D, Vester B, Fastinger N, and Swanson KS. 2009. Dietary protein concentration affects intestinal principle of energy minimization. Journal of Animal Ecology:411-432. 
437 Moreno MF, Sarantinopoulos P, Tsakalidou E, and De Vuyst L. 2006. The role and application of enterococci in food and health. International Journal of Food Microbiology 106:1-24.

Murray BE. 1990. The life and times of the Enterococcus. Clinical Microbiology Reviews 3:46-65.

Nesje M, Røed K, Lifjeld J, Lindberg P, and Steen O. 2000. Genetic relationships in the peregrine falcon (Falco peregrinus) analysed by microsatellite DNA markers. Molecular Ecology 9:53-60.

Nicholson JK, Holmes E, and Wilson ID. 2005. Gut microorganisms, mammalian metabolism and personalized health care. Nature Reviews: Microbiology 3:431.

O'Mara MT, Wikelski M, Voigt CC, Ter Maat A, Pollock HS, Burness G, Desantis LM, and Dechmann DKN. 2017. Cyclic bouts of extreme bradycardia counteract the high metabolism of frugivorous bats. eLife 6:e26686. https://doi.org/10.7554/eLife.26686.002

Omahony SM, Clarke G, Borre YE, Dinan TG, and Cryan JF. 2015. Serotonin, tryptophan metabolism and the brain-gut-microbiome axis. Behavioural Brain Research 277:32-48.

Orosz SE, and Lichtenberger M. 2011. Avian respiratory distress: etiology, diagnosis, and treatment. Veterinary Clinics of North America: Exotic Animal Practice 14:241-255.

Padilla J, Parejo J, Salazar J, Martínez-Trancón M, Rabasco A, Sansinforiano E, and Quesada A. 2009. Isolation and characterization of polymorphic microsatellite markers in lesser kestrel (Falco naumanni) and cross-amplification in common kestrel (Falco tinnunculus). Conservation Genetics 10:1357.

Park HJ, Lee SE, Kim HB, Isaacson R, Seo KW, and Song KH. 2015. Association of obesity with serum leptin, adiponectin, and serotonin and gut microflora in beagle dogs. Journal of Veterinary Internal Medicine 29:43-50.

459

Pearce DS, Hoover BA, Jennings S, Nevitt GA, and Docherty KM. 2017. Morphological and genetic factors shape the microbiome of a seabird species (Oceanodroma leucorhoa) more than environmental and social factors. Microbiome 5:146. 
461 Pepin J, Valiquette L, Alary M, Villemure P, Pelletier A, Forget K, Pepin K, and Chouinard D. 2004.

462

463

464

465

466

467

468

469

470

471

472

473

474

475

476

477

478

479

480

481

482

483

484

485
Clostridium difficile-associated diarrhea in a region of Quebec from 1991 to 2003: a changing pattern of disease severity. Canadian Medical Association Journal 171:466-472.

Qin J, Li R, Raes J, Arumugam M, Burgdorf KS, Manichanh C, Nielsen T, Pons N, Levenez F, and Yamada T. 2010. A human gut microbial gene catalogue established by metagenomic sequencing. Nature 464:59-65.

Riegert J, Fainová D, and Bystřická D. 2010. Genetic variability, body characteristics and reproductive parameters of neighbouring rural and urban common kestrel (Falco tinnuculus) populations. Population Ecology 52:73-79.

Schleucher E. 2002. Metabolism, body temperature and thermal conductance of fruit-doves (Aves: Columbidae, Treroninae). Comparative Biochemistry and Physiology Part A: Molecular \& Integrative Physiology 131:417-428. https://doi.org/10.1016/S1095-6433(01)00499-8

Scott KP, Gratz SW, Sheridan PO, Flint HJ, and Duncan SH. 2013. The influence of diet on the gut microbiota. Pharmacological Research 69:52-60.

Sela D, Chapman J, Adeuya A, Kim J, Chen F, Whitehead T, Lapidus A, Rokhsar D, Lebrilla CB, and German J. 2008. The genome sequence of Bifidobacterium longum subsp. infantis reveals adaptations for milk utilization within the infant microbiome. P Nati Acad Sci USA 105:1896418969.

Smit B, Zietsman G, Martin RO, Cunningham SJ, McKechnie AE, and Hockey PAR. 2016. Behavioural responses to heat in desert birds: implications for predicting vulnerability to climate warming. Climate Change Responses 3:9. https://doi.org/10.1186/s40665-016-0023-2

Souttou K, Baziz B, Doumandji S, Denys C, and Brahimi R. 2007. Prey selection in the common kestrel, Falco tinnunculus (Aves, Falconidae) in the Algiers suburbs (Algeria). Folia Zool 56:405.

Taylor MJ, Mannan RW, U'Ren JM, Garber NP, Gallery RE, and Arnold AE. 2019. Age-related variation in the oral microbiome of urban Cooper's hawks (Accipiter cooperii). BMC Microbiology 19:47.

Peer) reviewing PDF | (2020:01:45085:3:0:NEW 17 Aug 2020) 
486

487

488

489

491

492

493

494

495

496

497

498

499

500

501

502

503

504

505

506

507

508

509

510

511

Thoetkiattikul H, Mhuantong W, Laothanachareon T, Tangphatsornruang S, Pattarajinda V, Eurwilaichitr L, and Champreda V. 2013. Comparative analysis of microbial profiles in cow rumen fed with different dietary fiber by tagged 16S rRNA gene pyrosequencing. Current Microbiology 67:130137.

Turnbaugh PJ, Bäckhed F, Fulton L, and Gordon JI. 2008. Diet-induced obesity is linked to marked but reversible alterations in the mouse distal gut microbiome. Cell Host Microbe 3:213-223.

Turroni F, Bottacini F, Foroni E, Mulder I, Kim J-H, Zomer A, Sánchez B, Bidossi A, Ferrarini A, and Giubellini V. 2010. Genome analysis of Bifidobacterium bifidum PRL2010 reveals metabolic pathways for host-derived glycan foraging. P Nati Acad Sci USA 107:19514-19519.

van Dongen WF, White J, Brandl HB, Moodley Y, Merkling T, Leclaire S, Blanchard P, Danchin É, Hatch SA, and Wagner RH. 2013. Age-related differences in the cloacal microbiota of a wild bird species. BMC Ecology 13:11.

Van Zyl A. 1994. A comparison of the diet of the Common kestrel Falco tinnunculus in South Africa and Europe. Bird Study 41:127-130.

Village A. 2010. The kestrel: Bloomsbury Publishing.

Wang W, Zheng S, Sharshov K, Cao J, Sun H, Yang F, Wang X, and Li L. 2016. Distinctive gut microbial community structure in both the wild and farmed Swan goose (Anser cygnoides). Journal of Basic Microbiology 56:1299-1307.

Wang W, Zheng S, Sharshov K, Sun H, Yang F, Wang X, Li L, and Xiao Z. 2017. Metagenomic profiling of gut microbial communities in both wild and artificially reared Bar-headed goose (Anser indicus). MicrobiologyOpen 6:e0429.

Wilkinson TJ, Cowan A, Vallin H, Onime L, Oyama LB, Cameron S, Gonot C, Moorby J, Waddams K, and Theobald V. 2017. Characterization of the microbiome along the gastrointestinal tract of growing turkeys. Front Microbiol 8:1089.

Williams JB, and Tieleman BI. 2005. Physiological Adaptation in Desert Birds. Bioscience 55:416-425. https://doi.org/10.1641/0006-3568(2005)055[0416:PAIDB]2.0.CO;2

Peer] reviewing PDF | (2020:01:45085:3:0:NEW 17 Aug 2020) 
512 Winter M, Johnson DH, and Shaffer JA. 2006. Does body size affect a bird's sensitivity to patch size and

513 landscape structure? Condor 108:808-816. https://doi.org/10.1650/0010-

$514 \quad$ 5422(2006)108[808:DBSAAB]2.0.CO;2

515 Wu X, Zhang H, Chen J, Shang S, Yan J, Chen Y, Tang X, and Zhang H. 2017. Analysis and comparison

516 of the wolf microbiome under different environmental factors using three different data of Next

$517 \quad$ Generation Sequencing. Scientific Reports 7:11332.

518 Zhang L, Liu Y, and Song J. 2008. Genetic Variation Between Subspecies of Common Kestrels (Falco

519 Tinnunculus) in Beijing, China. J Raptor Res 42:214-220.

520 Zhao G, Zhou L, Dong Y, Cheng Y, and Song Y. 2017. The gut microbiome of hooded cranes (Grus

521 monacha) wintering at Shengjin Lake, China. MicrobiologyOpen 6:e00447.

522

523

524 
Figure 1

Sobs index and the Shannon index of samples
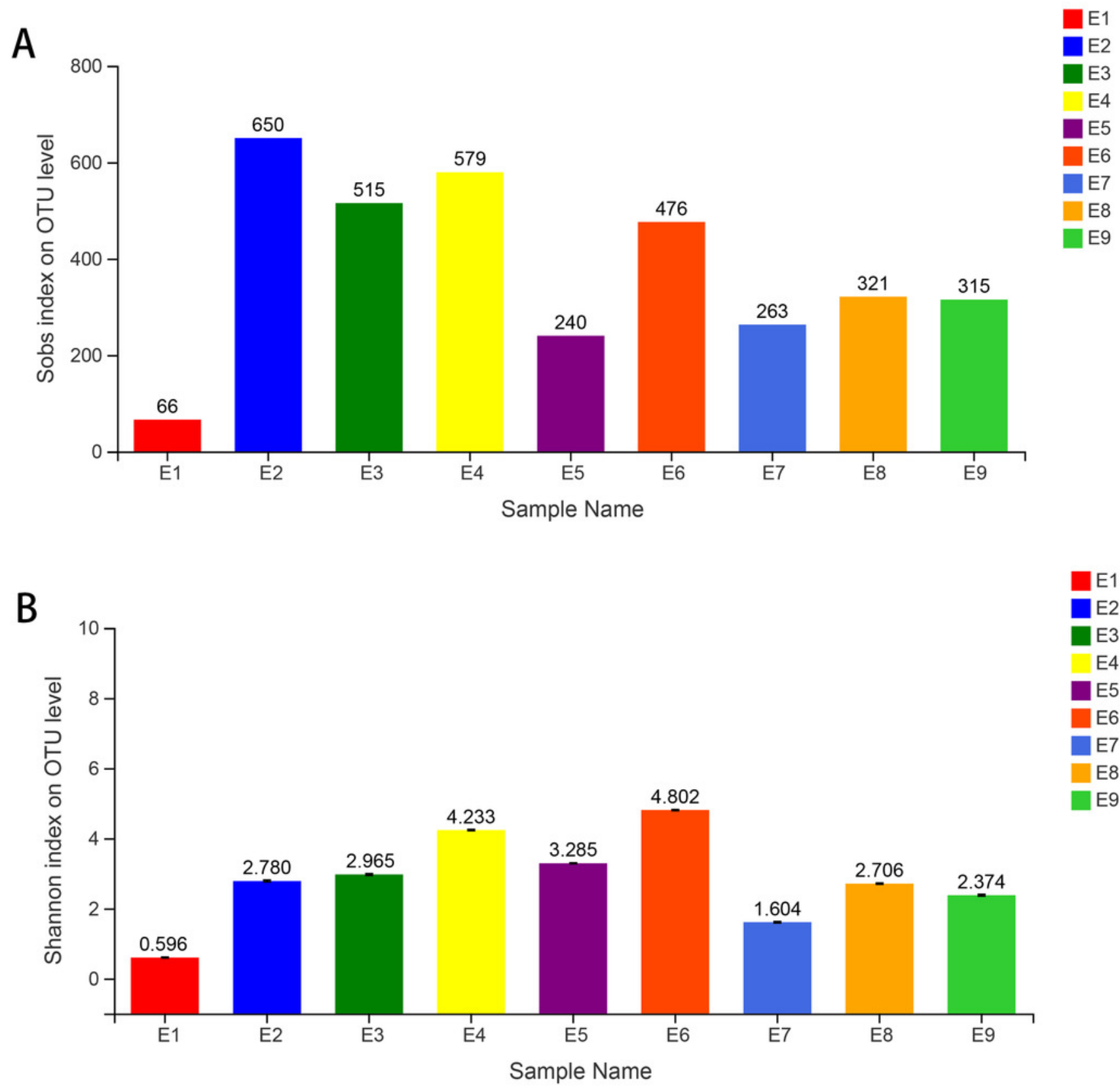
Figure 2

The histogram of relative abundance for species in Common Kestrel at phylum $(A)$ and genus (B) level.
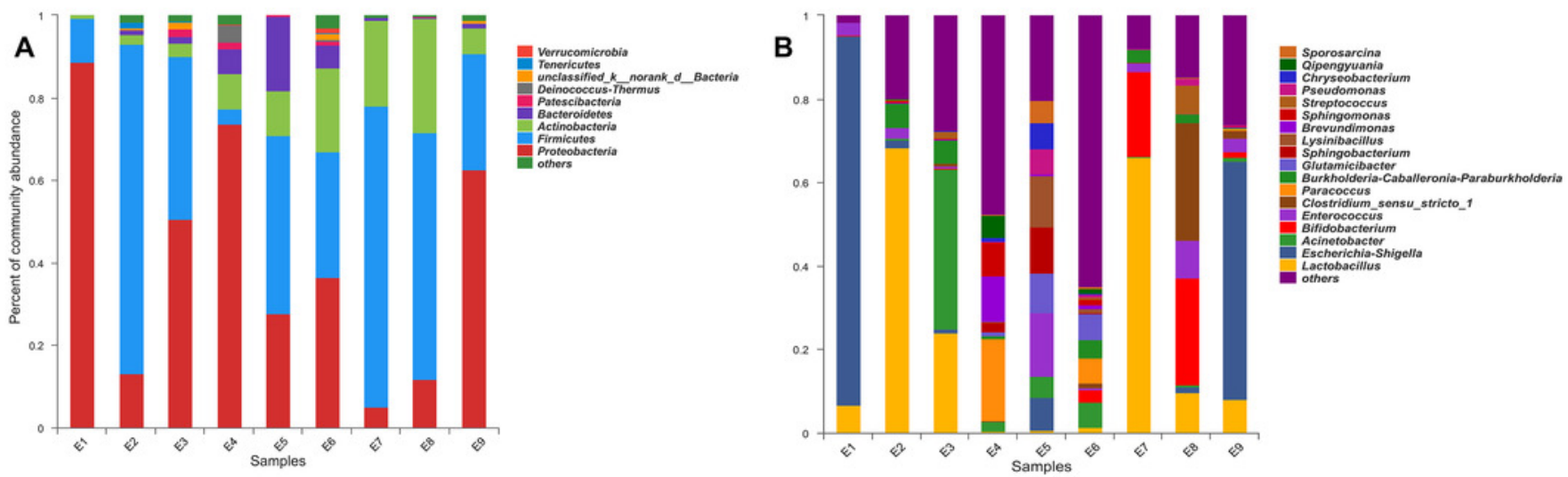
Figure 3

The hierarchical clustering trees

(A) and (B) were generated based on unweighted and weighted distance matrix at phylum level respectively. 


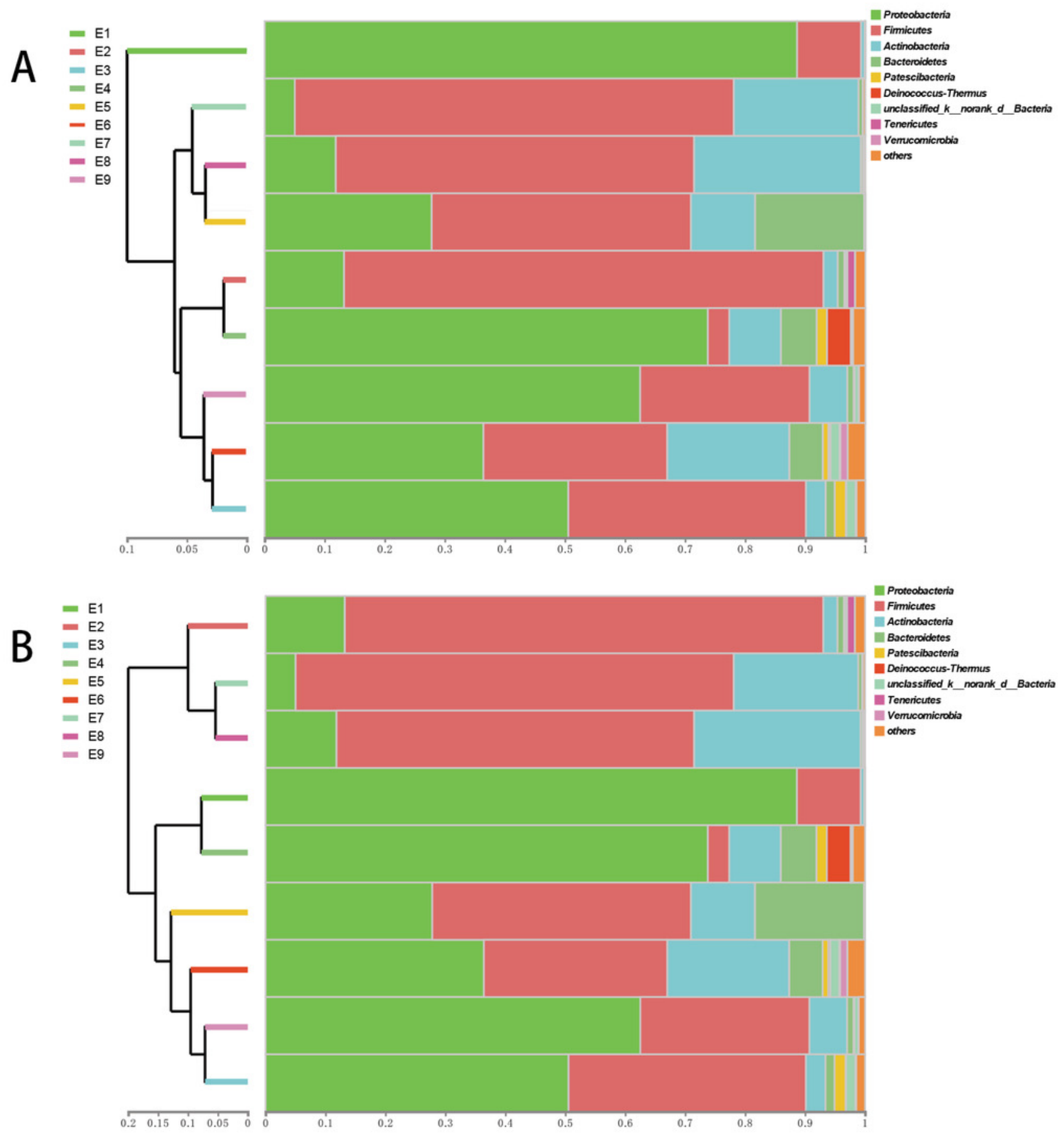


Figure 4

PCoA of the bacterial population structures.

The different shape with colors represented all samples of Common Kestrel respectively. For PCoA, (A) was generated with unweighted Unifrac distance while (B) used weighted Unifrac distance.
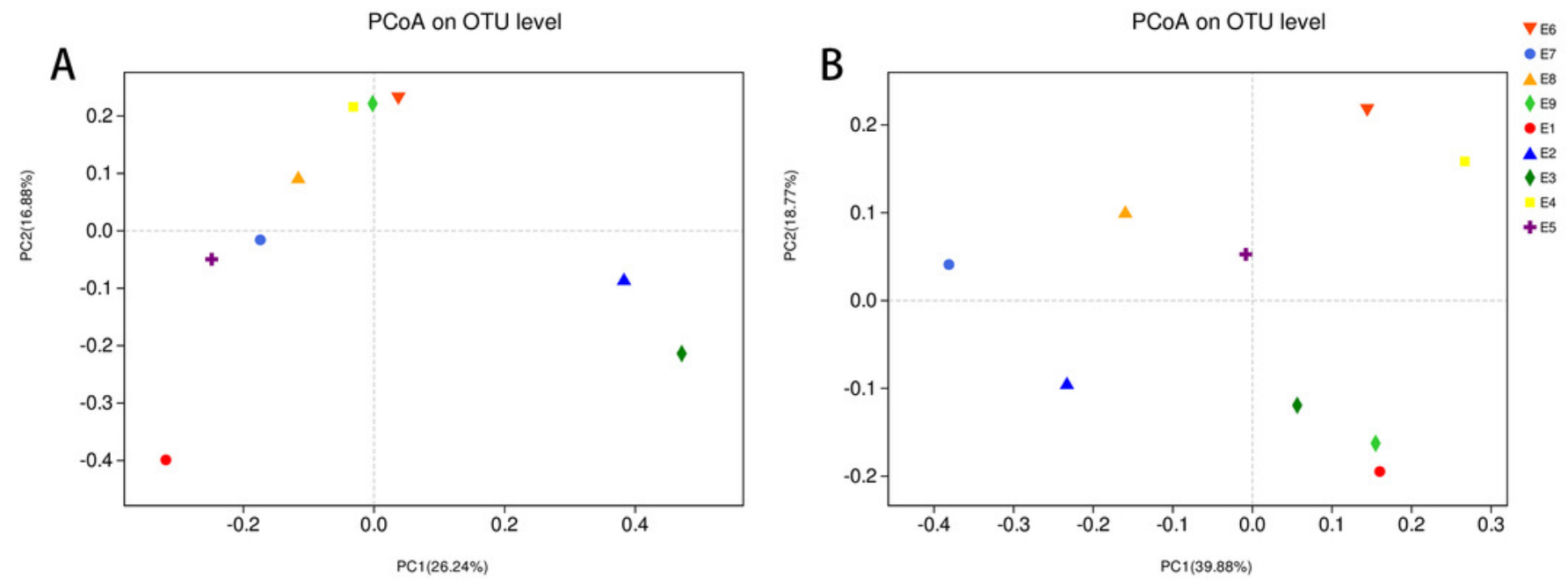


\section{Table 1 (on next page)}

Alpha diversity of gut microbiota in Common Kestrel feces 


\begin{tabular}{lllllll}
\hline Sample & Sobs & Shannon & Simpson & Ace & Chao & Coverage \\
\hline E1 & 66 & 0.596 & 0.788 & 78.114 & 73.583 & 1.000 \\
E2 & 649 & 2.780 & 0.204 & 674.412 & 672.193 & 0.998 \\
E3 & 515 & 2.965 & 0.184 & 524.452 & 522.519 & 0.999 \\
E4 & 578 & 4.233 & 0.053 & 594.498 & 594.050 & 0.999 \\
E5 & 235 & 3.285 & 0.057 & 448.368 & 378.103 & 0.997 \\
E6 & 476 & 4.802 & 0.020 & 479.110 & 480.091 & 1.000 \\
E7 & 263 & 1.604 & 0.399 & 292.553 & 281.800 & 0.999 \\
E8 & 317 & 2.706 & 0.143 & 364.651 & 359.519 & 0.998 \\
E9 & 317 & 2.374 & 0.335 & 330.906 & 331.607 & 0.999 \\
\hline
\end{tabular}




\section{Table 2 (on next page)}

The relative abundance of species in gut microbiota of Common Kestrel at phylum level

The names of phyla in Table 2 represented Proteobacteria, Firmicutes, Actinobacteria, Bacteroidetes, Patescibacteria, Deinococcus-Thermus, unclassified_K_norank_d_Bacteria, Tenericutes, Verrucomicrobia respectively. 


\begin{tabular}{|c|c|c|c|c|c|c|c|c|c|c|}
\hline Sample & Pro & Fir & Act & $B a c$ & Pat & Dei & unc & Ten & Ver & others \\
\hline E1 & $88.630 \%$ & $10.634 \%$ & $0.623 \%$ & $0.006 \%$ & $0.003 \%$ & $0.000 \%$ & $0.096 \%$ & $0.000 \%$ & $0.006 \%$ & $0.003 \%$ \\
\hline E2 & $13.211 \%$ & $79.816 \%$ & $2.376 \%$ & $1.085 \%$ & $0.085 \%$ & $0.065 \%$ & $0.361 \%$ & $1.291 \%$ & $0.017 \%$ & $1.694 \%$ \\
\hline E3 & $50.540 \%$ & $39.567 \%$ & $3.286 \%$ & $1.502 \%$ & $1.857 \%$ & $0.087 \%$ & $1.553 \%$ & $0.121 \%$ & $0.011 \%$ & $1.474 \%$ \\
\hline E4 & $73.770 \%$ & $3.574 \%$ & $8.602 \%$ & $5.950 \%$ & $1.719 \%$ & $3.960 \%$ & $0.220 \%$ & $0.008 \%$ & $0.158 \%$ & $2.038 \%$ \\
\hline E5 & $27.797 \%$ & $43.152 \%$ & $10.694 \%$ & $18.166 \%$ & $0.104 \%$ & $0.042 \%$ & $0.006 \%$ & $0.000 \%$ & $0.000 \%$ & $0.039 \%$ \\
\hline E6 & $36.410 \%$ & $30.610 \%$ & $20.330 \%$ & $5.572 \%$ & $0.944 \%$ & $0.324 \%$ & $1.511 \%$ & $0.135 \%$ & $1.223 \%$ & $2.940 \%$ \\
\hline E7 & $5.000 \%$ & $73.097 \%$ & $20.770 \%$ & $0.676 \%$ & $0.003 \%$ & $0.000 \%$ & $0.076 \%$ & $0.000 \%$ & $0.006 \%$ & $0.372 \%$ \\
\hline E8 & $11.832 \%$ & $59.652 \%$ & $27.752 \%$ & $0.369 \%$ & $0.073 \%$ & $0.003 \%$ & $0.031 \%$ & $0.000 \%$ & $0.031 \%$ & $0.256 \%$ \\
\hline E9 & $62.507 \%$ & $28.205 \%$ & $6.285 \%$ & $1.065 \%$ & $0.096 \%$ & $0.056 \%$ & $0.671 \%$ & $0.045 \%$ & $0.006 \%$ & $1.063 \%$ \\
\hline Mean & $41.078 \%$ & $40.923 \%$ & $11.191 \%$ & $3.821 \%$ & $0.543 \%$ & $0.504 \%$ & $0.503 \%$ & $0.178 \%$ & $0.162 \%$ & $1.098 \%$ \\
\hline
\end{tabular}

2 


\section{Table 3 (on next page)}

The relative abundance of species in gut microbiota of Common Kestrel at genus level

The names of phyla in Table 3 represented Lactobacillus, Escherichia-Shigella, Acinetobacter, Bifidobacterium, Enterococcus, Clostridium_sensu_stricto_1, Paracoccus, BurkholderiaCaballeronia-Paraburkholderia, Glutamicibacter respectively. 


\begin{tabular}{lllllllllll}
\hline Sample & Lac & Esc & Aci & Bif & Ent & Clo & Par & Bur & Glu & others \\
\hline E1 & $6.618 \%$ & $88.610 \%$ & $0.011 \%$ & $0.037 \%$ & $3.140 \%$ & $0.023 \%$ & $0.000 \%$ & $0.000 \%$ & $0.000 \%$ & $1.561 \%$ \\
E2 & $68.336 \%$ & $1.787 \%$ & $0.581 \%$ & $0.034 \%$ & $2.528 \%$ & $0.042 \%$ & $0.017 \%$ & $5.567 \%$ & $0.042 \%$ & $21.066 \%$ \\
E3 & $24.037 \%$ & $0.862 \%$ & $38.448 \%$ & $0.093 \%$ & $0.448 \%$ & $0.707 \%$ & $0.101 \%$ & $5.544 \%$ & $0.023 \%$ & $29.736 \%$ \\
E4 & $0.392 \%$ & $0.166 \%$ & $2.153 \%$ & $0.031 \%$ & $0.118 \%$ & $0.214 \%$ & $19.488 \%$ & $0.854 \%$ & $0.860 \%$ & $75.724 \%$ \\
E5 & $0.693 \%$ & $7.962 \%$ & $5.040 \%$ & $0.034 \%$ & $15.026 \%$ & $0.011 \%$ & $0.149 \%$ & $0.014 \%$ & $9.411 \%$ & $61.659 \%$ \\
E6 & $1.356 \%$ & $0.130 \%$ & $5.823 \%$ & $3.086 \%$ & $0.510 \%$ & $1.043 \%$ & $6.023 \%$ & $4.445 \%$ & $6.113 \%$ & $71.470 \%$ \\
E7 & $66.056 \%$ & $0.054 \%$ & $0.211 \%$ & $20.356 \%$ & $2.120 \%$ & $0.192 \%$ & $0.031 \%$ & $2.841 \%$ & $0.011 \%$ & $8.129 \%$ \\
E8 & $9.589 \%$ & $1.536 \%$ & $0.536 \%$ & $25.502 \%$ & $8.988 \%$ & $28.177 \%$ & $0.054 \%$ & $2.060 \%$ & $0.099 \%$ & $23.459 \%$ \\
E9 & $7.988 \%$ & $57.183 \%$ & $0.798 \%$ & $1.446 \%$ & $3.340 \%$ & $1.866 \%$ & $0.273 \%$ & $0.347 \%$ & $0.000 \%$ & $26.759 \%$ \\
Mean & $20.563 \%$ & $17.588 \%$ & $5.956 \%$ & $5.624 \%$ & $4.024 \%$ & $3.586 \%$ & $2.904 \%$ & $2.408 \%$ & $1.840 \%$ & $35.507 \%$ \\
\hline
\end{tabular}

